\title{
The role of moral disengagement and animal empathy in the meat paradox
}

\author{
Lauren Camilleri, Peter Richard Gill, Andrew Jago
}

\begin{abstract}
Current levels of meat consumption are environmentally unsustainable and cause suffering to billions of animals each year. Researchers have sought to understand the psychological mechanisms driving people's meat consumption. Research suggests that having empathy towards animals may reduce a person's meat consumption, however, an empathic response does not always result in action (the "meat paradox"). It may be that some people 'disengage' from their animal related morality when consuming meat. The current study aimed to test whether moral disengagement mediated the relationship between animal empathy and meat consumption. A total of 302 participants (18 to 70 years, $55.3 \%$ female) completed the Animal Empathy Scale, Moral Disengagement in Meat Questionnaire, and the Meat Consumption Scale. Results supported the mediation model, whereby high empathy for animals appeared to reduce one's capacity to disengage with their morality, resulting in lower meat consumption. This study was the first to investigate the dual role of animal empathy and moral disengagement in meat consumption. We conclude that moral disengagement is a possible explanation for the meat paradox.
\end{abstract}

Keywords: moral disengagement, animal empathy, meat consumption, meat paradox, gender differences 


\section{Introduction}

Global meat consumption is at an all-time high with approximately 56 billion landbased animals killed each year for food (Gullone, 2017). Research suggests that global meat consumption levels are unsustainable and result in environmental damage (Macdiarmid et al., 2016), inefficient use of natural resources (Pimentel \& Pimentel, 2003), mass-scale animal abuse (Mitchell, 2011), and health conditions such as heart disease (Neff et al., 2018) and cancer (Johnson, 2017). Currently, the mechanisms through which we might reduce meat consumption are unclear. Recent research suggests that empathy for animals may play a role in an individual's meat consumption behaviours.

\section{Meat consumption and animal empathy}

A recent meta-analysis of 155 studies identified that emotions and cognitive dissonance were the strongest individual predictors of meat consumption (Stoll-Kleemann \& Schmidt, 2017). Animal empathy is an emotional state of concern aroused by the suffering of an animal (Rothgerber \& Mican, 2014), and is distinct from human-directed empathy. While animal empathy can be understood as a stable trait, variations can occur depending on the type of animal concerned, and the context. Research suggests that greater animal empathy is associated with reduced meat consumption (Zickfeld et al., 2018). Research has also shown that when presented with images of animals in distress, vegetarians and vegans display greater activity in areas of the brain associated with empathy than meat eaters (Filippi et al., 2010). It appears that individuals high in empathy towards animals are more likely to make the connection between meat and its living animal origins, transforming an inanimate object on a plate into a living entity worthy of moral consideration. This moral consideration may prevent people from eating meat.

However, the relationship between animal empathy and meat consumption may be complex. As up to $97 \%$ of the Western population consumes meat and a similar percentage 
52 demonstrate animal empathy, then animal empathy does not always predict meat consumption. Some researchers have referred to this as the meat paradox, a psychological conflict that exists when people's empathy for animals and awareness of ethical and environmental problems with meat production, conflict with their enjoyment of eating meat (Bastian \& Loughnan, 2017). While some people resolve the meat paradox by eliminating or reducing their meat consumption, the majority do not. We suggest that moral disengagement may help explain the relationship between animal empathy and meat consumption.

\section{Rationale, Aims, Hypotheses}

In research on other prosocial behaviours, high empathy inhibits the activation of moral disengagement mechanisms, and motivates action, while low empathy enables moral disengagement mechanisms to operate, and decreases prosocial behaviour (Detert et al., 2008). It is possible that, in a similar process, animal empathy influences meat consumption indirectly, through moral disengagement. Strong feelings of animal empathy may inhibit the detachment and denial required to morally disengage, thereby providing motivation to alleviate the animal's suffering (i.e. reduce meat consumption). Alternatively, low levels of animal empathy would allow moral disengagement mechanisms to operate, resulting in increased meat consumption. This cognitive restructuring may reduce the dissonance associated with eating meat.

The current study will explore the potential mediating effect of moral disengagement on the relationship between animal empathy and meat consumption. Gender will also be investigated in relation to this mediation model, as research suggests that men consume more meat than women (Rothgerber \& Mican, 2014). It may be that moral disengagement has a stronger mediating effect for men than women. We will also test alternate mediation and 
moderation models in order to potentially rule them out. This is the first known study to investigate the role of moral disengagement in the meat paradox.

We hypothesise an indirect effect where high animal empathy will relate to lower moral disengagement with animals, and lower meat consumption. We also hypothesise that the mediating effect of moral disengagement will be stronger for men than women.

\section{Method}

\subsection{Participants and procedure}

Based on power analyses, researchers aimed to recruit at least 230 participants within a 3 month time frame. Following ethical approval, 346 Australian participants were recruited conveniently using Facebook and Twitter, and social networks, and participated via Qualtrics. Data from 302 participants (134 Male, 167 female, 1 unspecified) aged 18 to 70 years $(M=33.72, S D=12.71)$ were analysed. The majority of participants were single, living with housemates in a metropolitan/suburban area, working full-time, with an annual income below \$40K AUD. A large portion of participants had completed a bachelor or postgraduate degree. The proportion of vegetarians (9.3\%) and vegans (14.9\%) were greater than expected in the general population. All variables measured, and all analyses conducted are reported here.

\subsection{Measures}

Meat consumption. A meat consumption questionnaire was created. Participants were asked how frequently they consumed beef, lamb, chicken, seafood, ham/pork, and processed meats in the past two-week period. An 8-point Likert scale was used where $0=$ Never and $7=$ Multiple times a day. A meat consumption total score was the sum of the 6 meat items (range $=0$ to $42, \alpha=.83)$. 
98 Animal empathy. The Animal Empathy Scale (Paul, 2000) has 22 items and is scored on a 9-

99 point Likert scale (range $=0$ to 176 ). The scale has been reported to have adequate reliability

$100(\alpha=.78 ;$ Paul, 2000), and here $(\alpha=.76)$.

101 Moral disengagement. The 20 item Moral Disengagement in Meat Questionnaire (MDMQ)

102 (Graça et al., 2016) is scored on a 5-point Likert scale. The MDMQ has a global scale ( $\alpha=$

$103.93)$ and five subscales: means-ends justifications $(\alpha=.86)$, desensitisation $(\alpha=.90)$, denial

104 of negative consequences $(\alpha=.89)$, diffused responsibility $(\alpha=.84)$, and reduced perceived

105 choice $(\alpha=.70)$.

106

\subsection{Design and statistical analysis}

107 A cross-sectional correlational design was utilised. Simple mediation analysis was

108 conducted using Hayes' (2018) PROCESS Model 4 (with 5000 bootstrap samples) to

109 investigate the indirect path (see Figure 1). Mediation occurs when the 95\% CI of the indirect

110 effect does not contain zero.

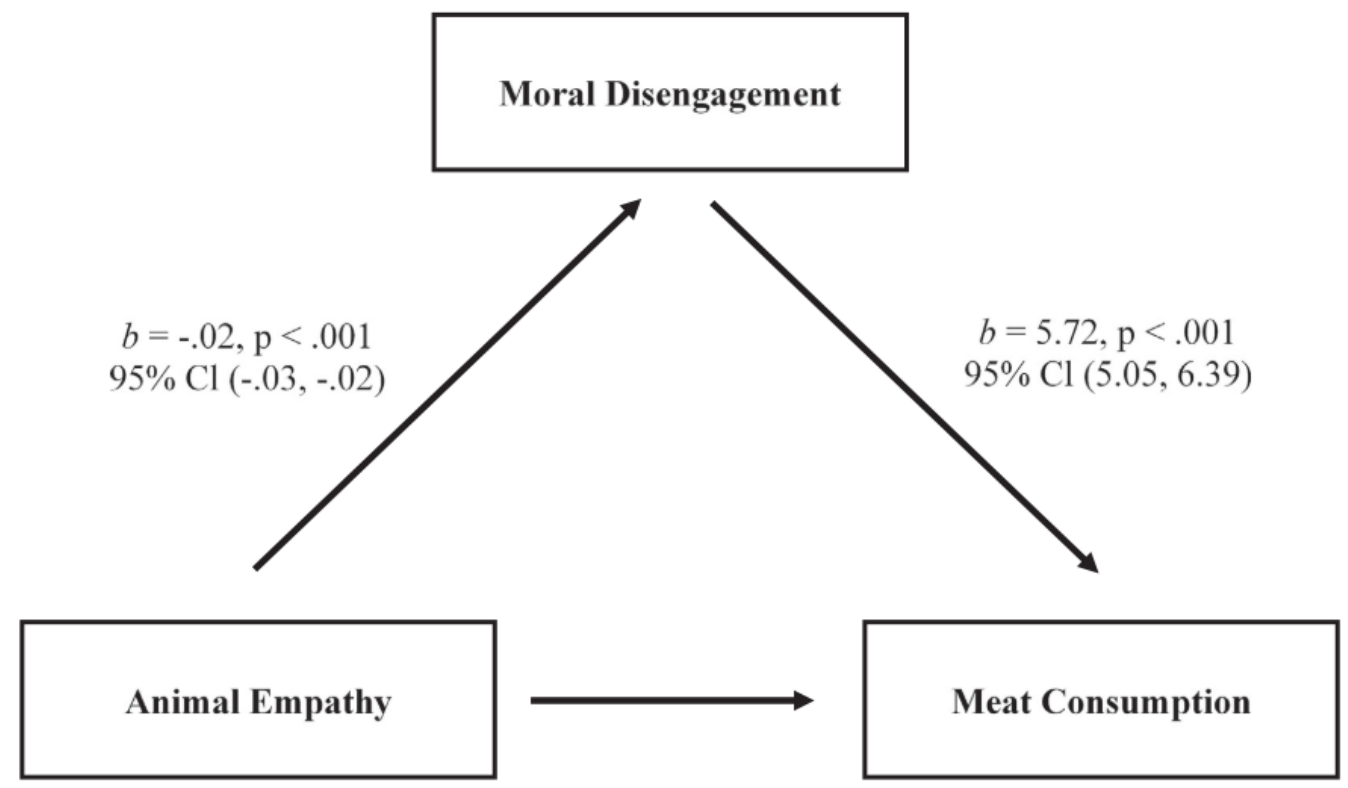

Direct effect, $b=.02, \mathrm{p}=.32,95 \% \mathrm{Cl}(-.02, .05)$

Indirect effect, $b=-.13, \mathrm{p}<.001,95 \% \mathrm{Cl}(-.16,-.11)$ 
An alternate mediation path and a moderation model were tested, where animal

113 empathy mediates the moral disengagement/ meat consumption relationship, and where

114 moral disengagement moderates the animal empathy/ meat consumption relationship.

115 Moderation occurs when the interaction variable 95\% CI does not contain zero.

116 Gender was investigated as a moderator of the relationships between animal empathy

117 and moral disengagement, and moral disengagement and meat consumption (see Figure 2),

118 Model 58 (5000 bs).

Overall:

$b=-.01(-.02,-.001)$,

$\mathrm{p}<.001$

Men:

$b=-.015(-.02,-.01)$

Women:

$b=-.024(-.03,-.02)$

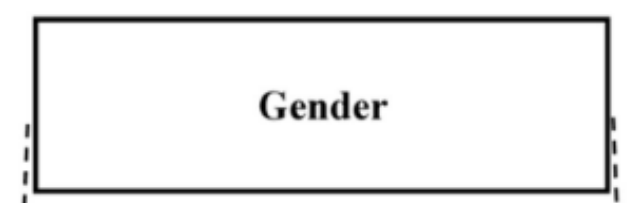

$b=.42(-.82,1.65), \mathrm{p}$

\section{Moderator}

$$
=.51
$$

$b=-.02(-.03,-.02)$

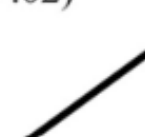

$\gamma$

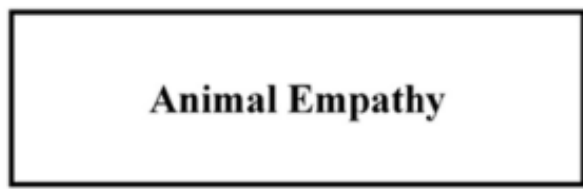

IV

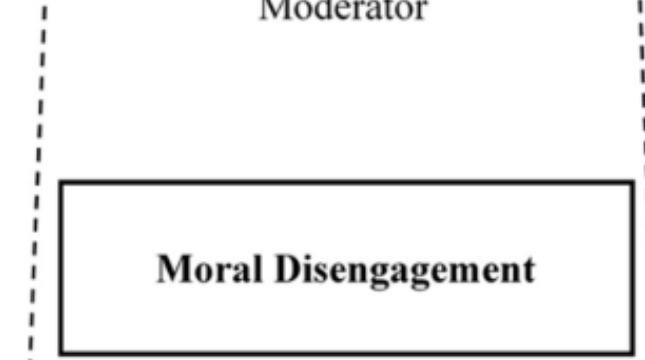

$b=5.72(5.05,6.39)$
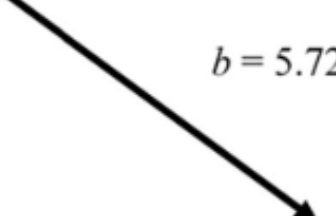

\section{Meat Consumption}

DV 


\section{Results}

121

122

123

124

125

126

127

128

130

131

132

133

134 3

\subsection{Descriptive statistics}

There were no violations of assumptions. Descriptive statistics for meat consumption, animal empathy, moral disengagement and the five moral disengagement subscales, and t-test results are presented in Table 1. Men consumed significantly more meat than women, had significantly higher moral disengagement, and had significantly lower empathy.

\subsection{Mediation tests}

As shown in figure 1, when the mediator was included in the model, the direct effect of animal empathy on meat consumption was no longer significant, and the indirect effect of animal empathy on meat consumption through moral disengagement total score (path $a b$ ) was significant as zero was not in the CI.

A series of mediation analyses performed separately for each of the five MDMQ subscales were all supportive of mediation with similar effect sizes; Means-ends justifications $b$ $=-.08$, Desensitisation $b=-.08$, Denial of negative consequences $b=-.06$, Diffused responsibility $b=-.05$, Reduced perceived choice $b=-.07$.

\subsection{Alternate mediation and moderation paths}

The indirect effect of moral disengagement on meat consumption through animal empathy (path $a b$ ) was not significant. No significant interaction was found between animal empathy and moral disengagement on meat consumption.

(1)

(1) 
144

145

\begin{tabular}{|c|c|c|c|c|c|c|c|c|}
\hline & \multicolumn{2}{|c|}{$\operatorname{Men}^{\mathrm{a}}$} & \multicolumn{2}{|c|}{ Women $^{\mathrm{b}}$} & \multirow[b]{2}{*}{$t$} & \multirow[b]{2}{*}{$p$} & \multicolumn{2}{|c|}{ Total $^{\mathrm{c}}$} \\
\hline & $M$ & $(S D)$ & $M$ & $(S D)$ & & & $M$ & $(S D)$ \\
\hline Meat Consumption & 18.43 & $(5.70)$ & 13.16 & $(5.63)$ & 8.03 & $<.001$ & 15.51 & $(6.22)$ \\
\hline Animal Empathy & 130.1 & $(17.65)$ & 145.57 & $(16.95)$ & 7.73 & $<.001$ & 138.68 & $(18.85)$ \\
\hline Moral Disengagement & 2.69 & $(0.72)$ & 2.04 & $(0.80)$ & 7.26 & $<.001$ & 2.33 & $(0.83)$ \\
\hline Means-ends Justifications & 3.08 & $(1.02)$ & 2.50 & $(1.06)$ & 4.80 & $<.001$ & 2.76 & $(1.08)$ \\
\hline Desensitisation & 3.03 & $(1.23)$ & 1.91 & $(1.14)$ & 8.20 & $<.001$ & 2.42 & $(1.31)$ \\
\hline Denial of negative & 2.06 & $(0.87)$ & 1.70 & $(0.86)$ & 3.59 & $<.001$ & 1.87 & $(0.88)$ \\
\hline \multicolumn{9}{|l|}{ consequences } \\
\hline Diffused Responsibility & 2.63 & $(0.92)$ & 2.06 & $(0.93)$ & 5.24 & $<.001$ & 2.31 & $(0.97)$ \\
\hline Reduced perceived choice & 2.61 & $(1.08)$ & 1.97 & $(1.08)$ & 5.10 & $<.001$ & 2.56 & $(1.12)$ \\
\hline
\end{tabular}

146 Note. ${ }^{\mathrm{a}} n=134 .{ }^{\mathrm{b}} n=167 .{ }^{\mathrm{c}} n=302$. 


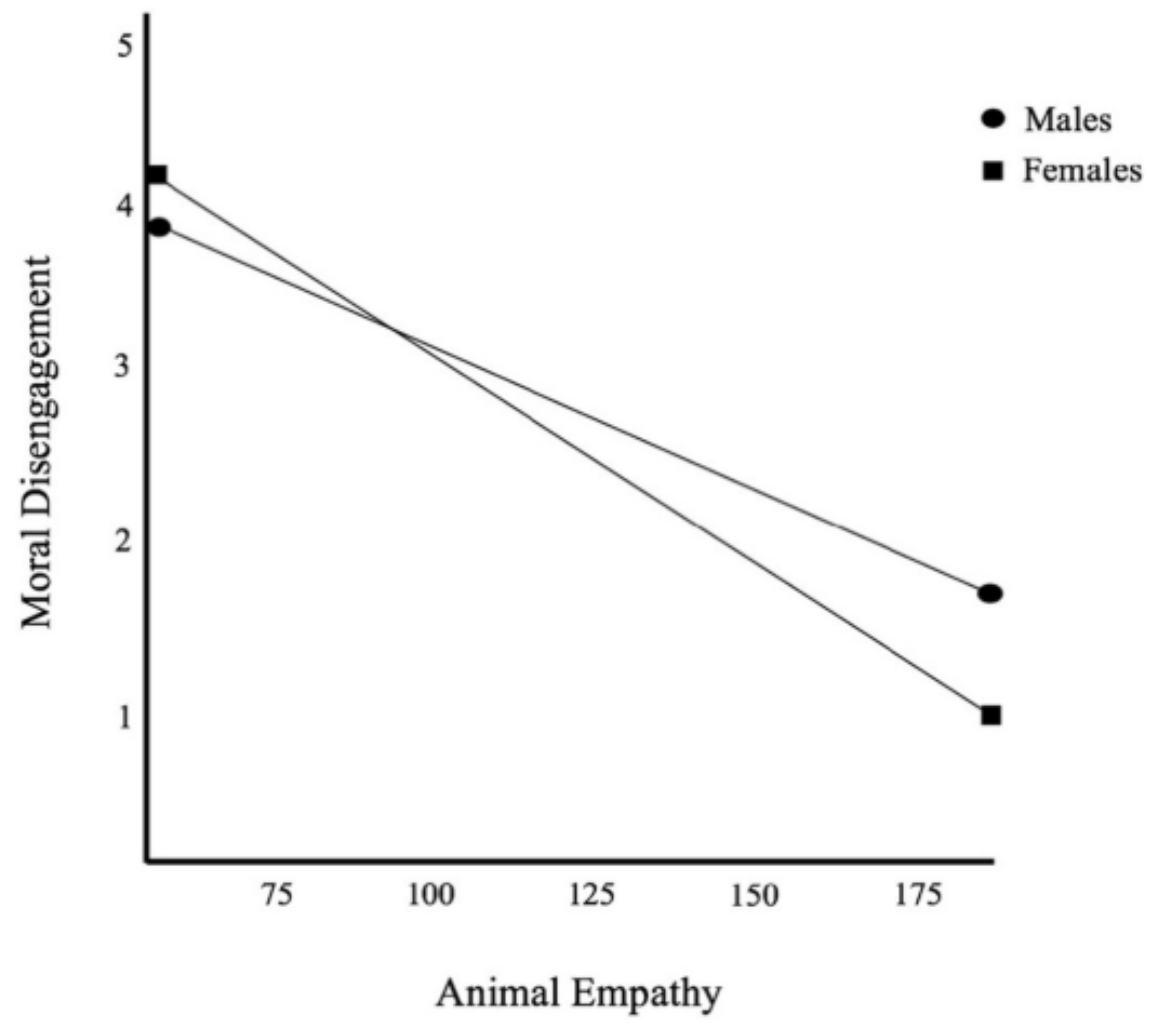

Fig. 3. Significant interaction between animal empathy and gender in relation to moral disengagement.

\section{Discussion}

157 The results supported the mediation model, where low animal empathy was associated with

158 higher levels of moral disengagement and greater meat consumption. No alternative models

159 fit the data. All moral disengagement subscales were found to significantly mediate the

160 relationship between animal empathy and meat consumption, showing the same pattern of 
161 low empathy $\rightarrow$ high moral disengagement $\rightarrow$ high meat consumption. The mediating effect was consistent across subscales. The current study's findings are consistent with previous mediation studies involving other prosocial behaviours (Detert et al., 2008). These findings

164 highlight the importance of moral disengagement in the relationship between animal empathy

165 and meat consumption. The degree to which a person morally disengages may be a key

166 determinant in meat consumption. Due to a cross-sectional design, causal inferences cannot be made. We cannot be certain whether animal empathy inhibits moral disengagement or vice versa. As research suggests that empathy inhibits the activation of moral disengagement (Paciello et al., 2013), it may be that a person who empathises strongly with the suffering of an animal is less able to deny the harm caused to it. As such, moral disengagement mechanisms, which serve to deny harm, are negated. Alternatively, moral disengagement may have an inhibiting effect on animal empathy, such that automatic empathetic responses are suppressed by moral disengagement mechanisms. It is possible that a person may initially experience animal empathy, but that moral disengagement mechanisms suppress the empathetic response. Given that eating meat is a largely unquestioned social norm embedded in daily life, it can be argued that moral disengagement mechanisms function as a stable disposition over time, rather than activating only under specific circumstances. It would therefore require strong empathetic arousal to override these socially conditioned default mechanisms. As the

180 alternate mediation model was not significant, the current study's findings suggest that 181 animal empathy has an inhibiting effect on moral disengagement. Specifically, higher empathy might be related to decreased tendencies to dehumanise and deny the suffering of animals, and to obviate responsibility for these outcomes (Buttlar \& Walther, 2018).

184 Longitudinal research is needed to establish the direction of causality among variables. 
influence on moral disengagement among women, providing a possible explanation for may be that due to gender-role socialisation, animal empathy has a more powerful inhibiting

190 effect on moral disengagement for women, which in turn, lowers their meat consumption.

191 Greater comparative female meat consumption might be the cumulative effect of both stronger empathy and a stronger effect of empathy on moral disengagement.

\section{Limitations} purposes specifically. A person may feel less empathy towards animals slaughtered for food than other animals. The measure of meat consumption used requires further validation. The sample had a greater proportion of vegetarians and vegans than in the general population.

\section{Conclusion}

It appears that moral disengagement mediates the relationship between animal empathy and meat consumption. These results suggest that when designing psychosocial strategies to reduce global meat consumption we should focus on ways of reducing moral disengagement, either through increased animal empathy (possibly more effective for women), or through other mechanisms. The mediating role of moral disengagement provides a possible part explanation for the meat paradox. 
210

211

212

213

214

215

216

217

218

219

220

221

222

223

224

225

226

227

228

229

230

231

232

233

234

\section{References}

Bastian, B., \& Loughnan, S. (2017). Resolving the meat-paradox: A motivational account of morally troublesome behavior and its maintenance. Personality and Social Psychology Review, 21(3), 278-299. https://doi.org/10.1177/1088868316647562

Buttlar, B., \& Walther, E. (2018). Measuring the meat paradox: How ambivalence towards meat influences moral disengagement. Appetite, 128, 152-158. https://doi.org/10.1016/j.appet.2018.06.011

Detert, J. R., Trevino, L. K., \& Sweitzer, V. L. (2008). Moral disengagement in ethical decision making: A study of antecedents and outcomes. Journal of Applied Psychology, 93(2), 374-391. https://doi.org/10.1037/0021-9010.93.2.374

Filippi, M., Riccitelli, G., Falini, A., Di Salle, F., Vuilleumier, P., Comi, G., \& Rocca, M. A. (2010). The brain functional networks associated to human and animal suffering differ among omnivores, vegetarians and vegans. PLOS ONE, 5(5), e10847. doi:10.1371/journal.pone.0010847

Graça, J., Calheiros, M. M., \& Oliveira, A. (2016). Situating moral disengagement: Motivated reasoning in meat consumption and substitution. Personality and Individual Differences, 90, 353-364. https://doi.org/10.1016/j.paid.2015.11.042

Gullone, E. (2017). Why eating animals is not good for us. Journal of Animal Ethics, 7(1), 31-62. http://www.press.uillinois.edu/journals/jane.html

Hayes, A. F. (2017). Introduction to mediation, moderation, and conditional process analysis $2^{\text {nd }}$ Ed. Guilford Press: NY.

Johnson, I. T. (2017). The cancer risk related to meat and meat products. British Medical Bulletin, 121, 73-81. https://doi.org/10.1093/bmb/ldw051

Macdiarmid, J., Douglas, F., \& Campbell, J. (2016). Eating like there's no tomorrow: Public awareness of the environmental impact of food and reluctance to eat less meat as part 
of a sustainable diet. Appetite, 96, 487-493. https://doi.org/10.1016/j.appet.2015.10.011

Mitchell, L. (2011). Moral disengagement and support for nonhuman animal farming. Society and Animals, 19, 38-58. https://doi.org/10.1163/156853011X545529

Neff, R. A., Edwards, D., Palmer, A., Ramsing, R., Righter, A., \& Wolfson, J. (2018). Reducing meat consumption in the USA: A nationally representative survey of attitudes and behaviours. Public Health and Nutrition, 21, 1835-1844. https://doi.org/10.1017/s1368980017004190

Paciello, M., Fida, R., Cerniglia, L., Tramontano, C., \& Cole, E. (2013). High cost helping scenario: the role empathy, prosocial reasoning and moral disengagement on helping behaviour. Personality and Individual Differences, 55, 3-7. https://doi.org/10.1016/j.paid.2012.11.004

Pimentel, D., \& Pimentel, M. (2003). Sustainability of meat-based and plant-based diets and the environment. American Journal of Clinical Nutrition, 78, 660S-663S. https://doi.org/10.1093/ajen/78.3.660S

Rothgerber, H., \& Mican, F. (2014). Childhood pet ownership, attachment to pets, and subsequent meat avoidance. The mediating role of empathy towards animals. Appetite, 79, 11-17. https://doi.org/10.1016/j.appet.2014.03.032

Stoll-Kleemann, S., \& Schmidt, U. J. (2017). Reducing meat consumption in developed and transition countries to counter climate change and biodiversity loss: a review of influence factors. Regional Environmental Change, 17(5), 1261-1277. doi:10.1007/s10113-016-1057-5

Zickfeld, J. H., Kunst, J. R., \& Hohle, S. M. (2018). Too sweet to eat: Exploring the effects of cuteness on meat consumption. Appetite, 120, 181-195. https://doi.org/10.1016/j.appet.2017.08.038 
260

261

262

263

264 